www.nature.com/pj

\title{
Improvement of the thermal stability of streptavidin immobilized on magnetic beads by the construction of a mixed poly(ethylene glycol) tethered-chain layer
}

\author{
Masaki Kubota $^{1}$, Keitaro Yoshimoto ${ }^{1,2,3}$, Xiaofei Yuan ${ }^{1,2,3}$ and Yukio Nagasaki ${ }^{1,2,3,4,5}$ \\ Polymer Journal (2011) 43, 493-496; doi:10.1038/pj.2011.5; published online 16 February 2011
}

Keywords: DNA; magnetic beads; PEG tethered-chain layer; poly(ethylene glycol) (PEG); streptavidin; thermal stability

\section{INTRODUCTION}

Protein-immobilized substrates have been widely used in several applications for biosensing and bioseparation. Many kinds of sensors have been based on changes in colorimetric, fluorometric or luminometric signals derived from the immunoreactions of protein-immobilized sensor chips and particles. ${ }^{1-4}$ Protein-immobilized substrates, such as column packings and magnetic beads, have also been used as selective materials that can rapidly and simply separate target biomolecules in solution.

In the development of high-performance materials for biosensing and bioseparation, an effective blocking treatment is important to decrease nonspecific adsorption onto the surfaces of protein-immobilized substrates and to increase the dispersion stability of substrate particles. Poly(ethylene glycol) (PEG) is known as an excellent blocking agent. Because of the nonionic properties, hydrophilicity and large steric-exclusion effect of PEG, ${ }^{5}$ PEGylated surfaces and nano- and microscale particles show excellent non-fouling properties with various molecules ${ }^{6}$ and high-dispersion stabilities, ${ }^{7-9}$ respectively. Furthermore, in our recent studies, we discovered that PEGylation improves the functioning of immobilized proteins on solid surfaces and particles. For example, the antigen-binding efficiencies of an antiC-reactive protein antibody-immobilized gold sensor surface ${ }^{10}$ and anti-ferritin antibody-immobilized latex particles ${ }^{11}$ were improved by the co-immobilization of densely packed PEG layers, consisting of sulfhydryl-terminated PEGs and oligoamine-terminated PEGs. The substrate reactivity of glucose dehydrogenase-immobilized gold nanoparticles was improved by the co-immobilization of PEG/polyamine block copolymer, ${ }^{8}$ and lipase/PEG-polyamine/glucose dehydrogenaseimmobilized gold nanoparticle hybrids showed almost the same initial enzymatic activity after five repeated thermal treatments at $58^{\circ} \mathrm{C}$ for 10 min. ${ }^{12}$ These results indicate that the co-immobilization of PEG derivatives onto protein-immobilized substrates has potential as a treatment to strongly improve the performance of biosensing and bioseparation materials.

The heat-induced inactivation of proteins is a disadvantage that often restricts their field of application. It is thought that heat-induced inactivation occurs because of the dissociation of intramolecular protein interactions. ${ }^{13}$ Here, we report the preventative effect of a densely packed PEG layer against the heat-induced inactivation of streptavidin-immobilized magnetic beads (SA-MB). SA-MB co-immobilized with a PEG tethered-chain layer (PEG/SA-MB) were constructed by the co-immobilization of oligoamine-terminated PEGs onto $\mathrm{SA}-\mathrm{MB}$, and their biotin-binding efficiency was evaluated by measuring the amount of biotinylated single-stranded DNA (ssDNA) captured by the modified SA-MB. We found that the capture efficiency of biotinylated ssDNA by PEG/SA-MB was twice that of unmodified SA-MB after repeated thermal treatments for $5 \mathrm{~min}$ at $75^{\circ} \mathrm{C}$.

\section{EXPERIMENTAL PROCEDURE}

Materials

Carboxylated magnetic beads (Magnosphere MS300/High Carboxyl, 10\% solid content in slurry, particle diameter $3 \mu \mathrm{m}$ ), $\alpha$-methoxy-PEG-pentaethylenehexamine (N6-PEG) at two different molecular weights, N6-PEG (5k) (Blockmaster CE510; $\left.M_{\mathrm{w}}=5000\right)$ and N6-PEG (2k) (Blockmaster CE210; $M_{\mathrm{w}}=2000$ ), were kind gifts from the JSR Corporation (Tokyo, Japan). Other chemicals were used as purchased. The sequence of the biotinylated ssDNA used in this study is 5'-(biotin)-ATAGGAGTCGACCGACCAGAA-3'.

\section{Construction of PEG/SA-MB and SA-MB}

Figure 1 shows a schematic illustration of the PEG/SA-MB. Streptavidin, ethanolamine and N6-PEGs were attached to the surface of carboxylated magnetic beads through the active ester reaction method in the same manner as previously reported. ${ }^{9111}$ Magnetic bead suspension $(10 \mu \mathrm{l})$ was poured into a

${ }^{1}$ Graduate School of Pure and Applied Science, University of Tsukuba, 1-1-1 Ten-noudai, Tsukuba, Ibaraki, Japan; ${ }^{2}$ Tsukuba Research Center for Interdisciplinary Materials Science (TIMS), University of Tsukuba, 1-1-1 Ten-noudai, Tsukuba, Ibaraki, Japan; ${ }^{3}$ Center for Tsukuba Advanced Research Alliance (TARA), University of Tsukuba, 1-1-1 Tennoudai, Tsukuba, Ibaraki, Japan; ${ }^{4}$ Master's School of Medical Science, Graduate School of Comprehensive Human Sciences, University of Tsukuba, 1-1-1 Ten-noudai, Tsukuba, Ibaraki, Japan and ${ }^{5}$ Satellite Laboratory, International Center for Materials Nanoarchitectonics (MANA), National Institute of Materials Science (NIMS), Tsukuba, Ibaraki, Japan Correspondence: Professor Y Nagasaki, Tsukuba Research Center for Interdisciplinary Materials Science (TIMS), University of Tsukuba, 1-1-1 Ten-noudai, Tsukuba, Ibaraki 305-8573, Japan.

E-mail: yukio@nagalabo.jp

Received 24 October 2010; revised and accepted 23 December 2010; published online 16 February 2011 


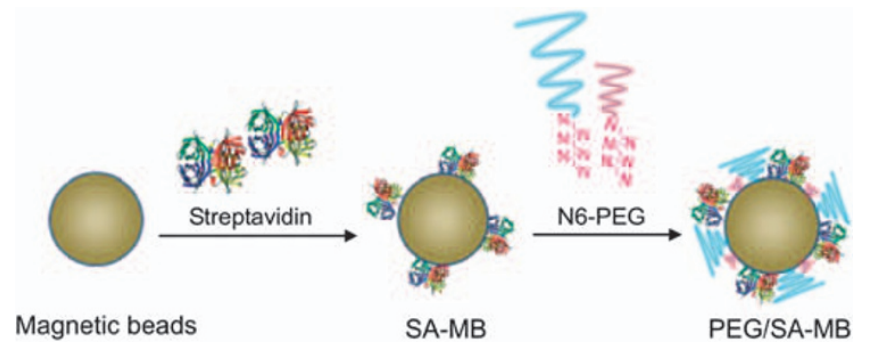

Figure 1 Schematic illustration of the preparation of streptavidinimmobilized magnetic beads (SA-MB) and poly(ethylene glycol) (PEG)/ streptavidin co-immobilized magnetic beads (PEG/SA-MB).

1.5-ml plastic tube, and the tube was placed on a magnetic separator to remove the supernatant. The remaining beads were dispersed into $1 \mathrm{ml}$ of $\mathrm{NaH}_{2} \mathrm{PO}_{4}$ buffer solution (10 mM, pH 4.7). Then $100 \mu \mathrm{l} \mathrm{of} 10 \mathrm{mg} \mathrm{ml}^{-1}$ 1-ethyl-3(3-dimethylaminopropyl)carbodiimide hydrochloride (EDC) solution (also in $\mathrm{NaH}_{2} \mathrm{PO}_{4}$ buffer) was added to this suspension, followed by shaking for $30 \mathrm{~min}$ at room temperature and at $\mathrm{pH}$ 4.7. After again removing the supernatant, using the magnetic separator, the beads were dispersed into 1-ml aliquots of streptavidin solution at various concentrations (again in $\mathrm{NaH}_{2} \mathrm{PO}_{4}$ buffer at $\mathrm{pH}$ 4.7). The reaction time for streptavidin immobilization was $15 \mathrm{~min}$ at room temperature. To construct a densely packed PEGylated surface, we used our original blocking procedure ${ }^{9-11,14-16}$ in this study, in which a mixed PEG tethered-chain layer was constructed on the SA-MB surface by the use of a mixture of long PEG (5k) and short PEG (2k), as follows: immediately after the immobilization of streptavidin, the beads were dispersed into $1 \mathrm{ml}$ of N6-PEG solution and incubated overnight. The N6-PEG solution was a mixture of $600 \mu \mathrm{l}$ of N6-PEG (5k) (1.5 wt\%, pH 7.4) and $400 \mu \mathrm{l}$ of N6-PEG (2k) (1.5 wt\%, $\mathrm{pH}$ 7.4) aqueous solutions. As control, unmodified SA-MB was prepared by treating streptavidin-conjugated beads with ethanolamine; the beads were dispersed into $1 \mathrm{ml}$ of ethanolamine aqueous solution $\left(6.8 \times 10^{-3} \mathrm{M}, \mathrm{pH} 7.4\right)$ and incubated overnight. The resulting beads were washed twice with phosphate-buffered saline (PBS) (10 mM sodium phosphate buffer containing $150 \mathrm{~mm} \mathrm{NaCl}, \mathrm{pH} 7.4$ ) and resuspended in $1 \mathrm{ml}$ of PBS.

\section{Measurement of the amount of biotinylated ssDNA captured by PEG/SA-MB and SA-MB}

To measure the amount of biotinylated ssDNA captured by PEG/SA-MB and SA-MB, the change in the absorbance of the ssDNA solution at $260 \mathrm{~nm}$ after bead incubation was measured using a NanoDrop 1000 (Thermo Fisher Scientific Inc., Waltham, MA, USA) as follows: bead suspension $(40 \mu \mathrm{l})$ was pipetted into a $0.2-\mathrm{ml}$ plastic tube and washed once with binding buffer $(10 \mathrm{~mm}$ Tris- $\mathrm{HCl}$ containing 0.5 mм EDTA, $1 \mathrm{M} \mathrm{NaCl}, 0.1$ wt $\%$ Tween-20, pH 7.4). Then the supernatant was removed and $10 \mu \mathrm{l}$ of biotinylated ssDNA solution $(5.0 \mu \mathrm{M}$, dissolved in binding buffer) was added. After incubation for $1 \mathrm{~h}$ at room temperature, the beads were separated and the absorbance of the supernatant at $260 \mathrm{~nm}$ was measured. The amounts of biotinylated ssDNA captured by PEG/SA-MB and SA-MB were estimated from the obtained absorbance, and the extinction coefficient of biotinylated ssDNA was estimated to be $2202001 \mathrm{~mol}^{-1} \mathrm{~cm}^{-1}$.

\section{Measurement of $\zeta$-potential}

The $\zeta$-potentials of PEG/SA-MB and SA-MB were measured on a Zetasizer Nano (Malvern Instruments Ltd, Worcestershire, UK). Measurements were taken at room temperature. All samples were dispersed into PBS, and the bead concentration was $0.05 \mathrm{mg} \mathrm{ml}^{-1}$.

\section{Evaluation of the amount of biotinylated ssDNA captured by the modified magnetic beads after heat treatment}

It was previously reported that the melting point of streptavidin is greater than $75^{\circ} \mathrm{C}$ at neutral $\mathrm{pH} .{ }^{13}$ Therefore, we used heat treatment at $75^{\circ} \mathrm{C}$ in this study. Before the incubation of the magnetic beads with the biotinylated ssDNA solutions, PEG/SA-MB and SA-MB were heated in an iCycler (Bio-Rad

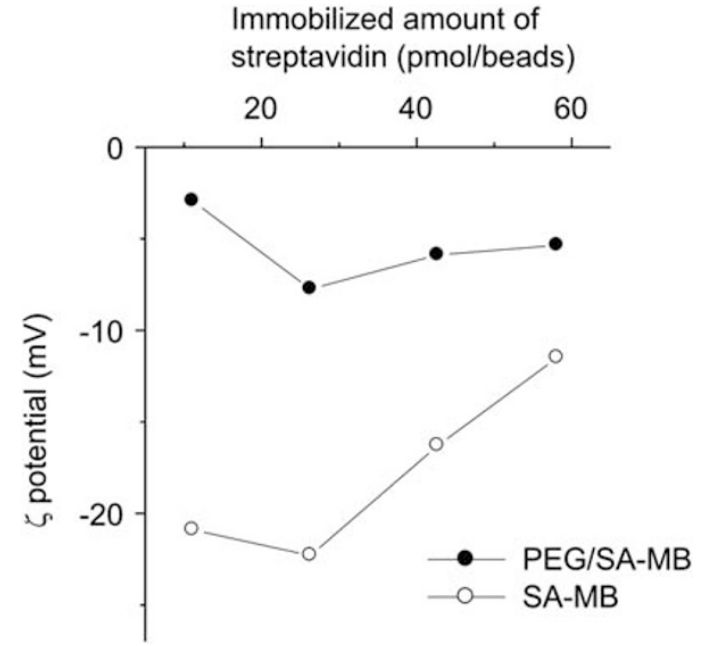

Figure 2 Changes in the $\zeta$-potentials of $(\bullet)$ poly(ethylene glycol) (PEG)/ streptavidin co-immobilized magnetic beads (PEG/SA-MB) and $(O)$ streptavidin-immobilized magnetic beads (SA-MB) according to the amount of immobilized streptavidin. All samples were dispersed in $10 \mathrm{~mm}$ sodium phosphate buffer containing $150 \mathrm{~mm} \mathrm{NaCl}$ at $\mathrm{pH} 7.4$, and measurements were taken at room temperature. Concentration of the bead was $0.05 \mathrm{mg} \mathrm{ml}^{-1}$.

Laboratories, Inc., Hercules, CA, USA). One to three cycles of heat treatment for $5 \mathrm{~min}$ at $75^{\circ} \mathrm{C}$ were performed. The estimation of the amount of biotinylated ssDNA captured by the modified magnetic beads was performed using the same procedures as described above. The same evaluation was performed in methoxy-PEG-OH (10 wt $\left.\%, M_{\mathrm{w}}=5000\right)$ containing PBS buffer.

\section{RESULTS AND DISCUSSION}

The immobilization of streptavidin on the magnetic bead (SA-MB) surfaces was performed by the active ester method. The functionality of the obtained SA-MB was estimated by the amount of biotinylated ssDNA captured. The quantitative data are listed in Supplementary Figure S1 in the Supplementary Information section. As shown here, the amount of biotinylated ssDNA captured by SA-MB increased with increasing streptavidin concentration in the immobilization reaction. These results indicate that the effective immobilization of streptavidin onto the magnetic beads was accomplished under the experimental conditions, and the amount of immobilized streptavidin can be controlled by changing the streptavidin concentration in the solution.

The immobilization of PEG in the form of a tethered-chain layer on the SA-MB surfaces was performed using N6-PEG, which has six amino groups at the end of each PEG chain. Although a monoamine end group is theoretically sufficient to conjugate a surface-active ester moiety, it is experimentally difficult to conjugate desirable amounts of PEG molecules because of the steric repulsion between the PEG chains. Because the SA-MB surface is negatively charged, the oligoamine moiety tends to access the SA-MB surface. This electrostatic attractive force facilitates the reaction of N6-PEG with the active ester moiety and the formation of a polyion complex with the liberated carboxylic acids on the surface of the magnetic beads. To increase the chain density, a mixed PEG tethered-chain layer was constructed on the SA-MB surfaces.

The amount of biotinylated ssDNA captured by the SA-MB was slightly higher than that captured by PEG/SA-MB (Supplementary Figure S2). To confirm the elaboration of the PEG layer on the magnetic beads, $\zeta$-potential analysis and observation of the dispersion stability in cell lysis buffer were performed. Figure 2 shows the changes 
in the $\zeta$-potential of PEG/SA-MB and SA-MB in relation to the amount of immobilized streptavidin. The $\zeta$-potential of the SA-MB increased with increasing amounts of streptavidin immobilized on the bead surface. For example, the $\zeta$-potential of SA-MB possessing $11.1 \mathrm{pmol}$ streptavidin per mg beads was $-20.9 \mathrm{mV}$, and it increased with increasing amounts of immobilized streptavidin, finally reaching

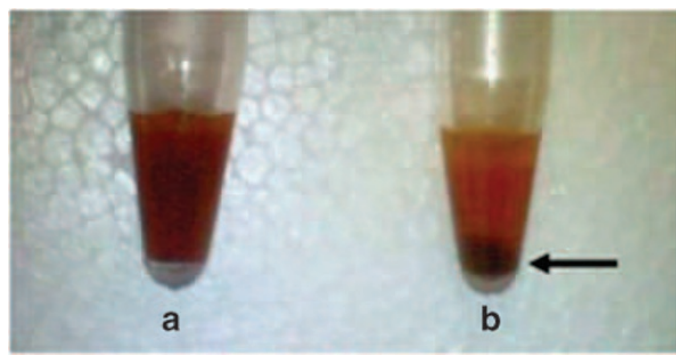

Figure $\mathbf{3}$ Images of (a) poly(ethylene glycol) (PEG)/streptavidin coimmobilized magnetic beads (PEG/SA-MB) and (b) streptavidin-immobilized magnetic beads (SA-MB) dispersed in $100 \mu \mathrm{l}$ of $5 \times$ cell lysis buffer $(\mathrm{pH}$ 8.0). The amount of streptavidin immobilized on these beads was $26.4 \mathrm{pmol}$ per mg beads, and 2-mg samples of each type of bead were incubated for $1 \mathrm{~h}$ at room temperature. The aggregation is indicated by arrow.

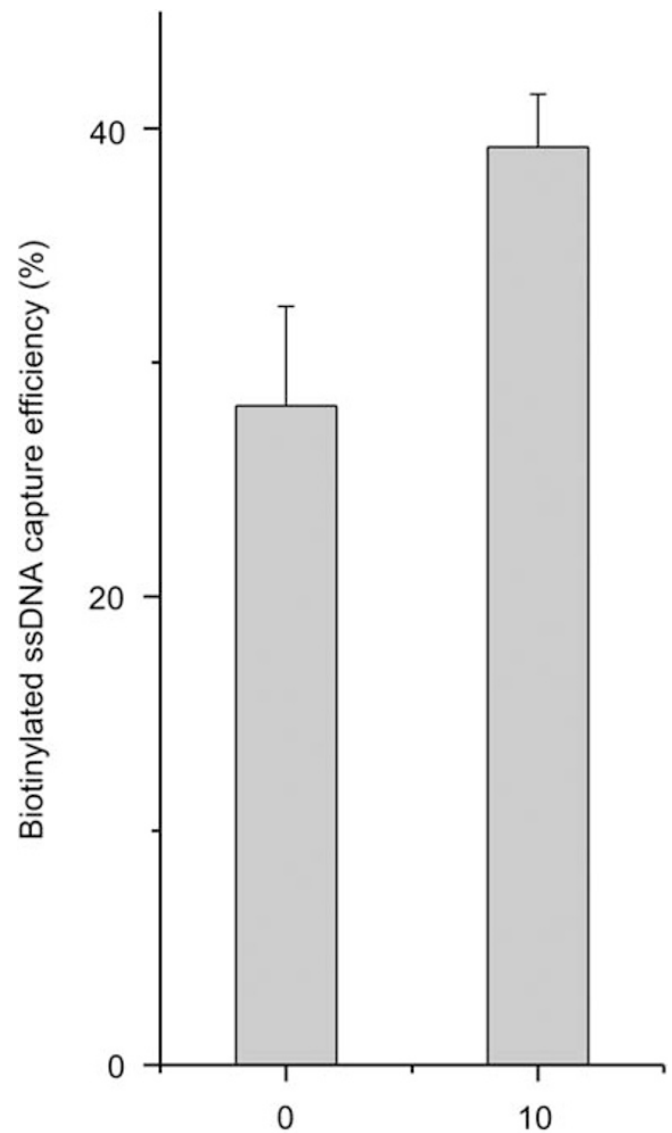

PEG concentration (wt\%)

Figure 4 The capture efficiency of biotinylated single-stranded DNA by streptavidin-immobilized magnetic beads (SA-MB) (with $26.4 \mathrm{pmol}$ of immobilized streptavidin per $\mathrm{mg}$ beads) after three cycles of heat treatment for $5 \mathrm{~min}$ at $75^{\circ} \mathrm{C}$ with and without $10 \mathrm{wt} \%$ poly(ethylene glycol) (PEG) (5k) in phosphate-buffered saline buffer. The efficiency was defined as the percentage of the initial value (before heat treatment).
$-11.5 \mathrm{mV}$. Because the isoelectric point of streptavidin is $6.8-7.5$, it is slightly negatively charged under these conditions. In contrast, the $\zeta$-potential of the PEG/SA-MB was approximately $-5 \mathrm{mV}$, regardless of the amount of immobilized streptavidin, indicating that the negatively charged surfaces of the magnetic beads were shielded almost completely by the PEG layer. Additionally, the dispersion stability of the modified beads was also evaluated. Figure 3 shows images of SA$\mathrm{MB}$ and PEG/SA-MB suspensions in cell lysis buffer containing nonionic and zwitterionic surfactants. As shown here, most of the SA-MB was precipitated after $1 \mathrm{~h}$, whereas no precipitation was observed from the PEG/SA-MB suspension. These results indicate that a PEG layer had formed effectively on the SA-MB surfaces.

Before the investigation of PEG co-immobilization on the SA-MB surfaces, we examined the effect of free PEG in the buffer solution on the stabilization of streptavidin on the magnetic bead surfaces. Free PEG stabilizes both protein structure and protein activity. For example, free PEG in the reaction medium functions as a stabilizer of penicillin acylase and Ca-ATPase. ${ }^{17,18}$ The flexibility and hydration of PEG is thought to help stabilize the compact structures of surrounded proteins because of increasing intramolecular hydrophobic interactions. ${ }^{19}$ Figure 4 shows the changes in the capture efficiency of biotinylated ssDNA by SA-MB after three cycles of heat treatment at $75^{\circ} \mathrm{C}$ in PBS, with and without a sufficient concentration (10 wt $\left.\%\right)$ of methoxy-PEG-OH (5k). The capture efficiency is here defined as the percentage of the initial capture amount (before heat treatment). In this experiment, SA-MB possessing $26.4 \mathrm{pmol}$ streptavidin per mg beads were used. When SA-MB were subjected to three cycles of heat treatment in PBS without PEG addition, the capture efficiency of biotinylated ssDNA was drastically reduced, reaching $29 \%$ of the initial capture amount. In the presence of $10 \mathrm{wt} \%$ commercial PEG, on the

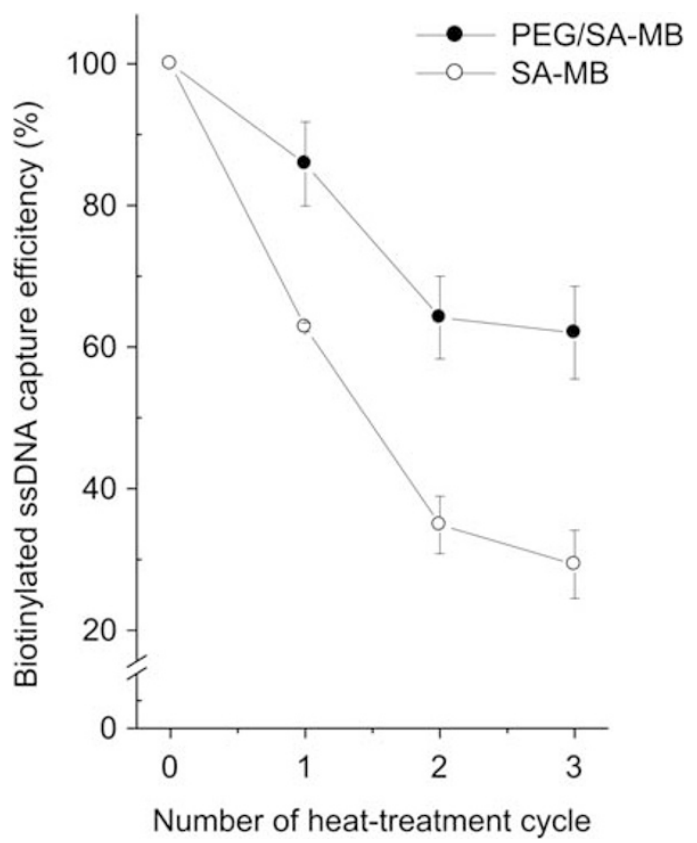

Figure 5 Changes in the capture efficiency of biotinylated single-stranded DNA by poly(ethylene glycol) (PEG)/streptavidin co-immobilized magnetic beads $(\mathrm{PEG} / \mathrm{SA}-\mathrm{MB})(\bullet)$ and by streptavidin-immobilized magnetic beads $(\mathrm{SA}-\mathrm{MB})(\mathrm{O})$ as a function of the number of heat-treatment cycles. The efficiencies were defined as percentages of the initial values (before heat treatment). The amount of streptavidin immobilized on both the PEG/SA-MB and SA-MB was $26.4 \mathrm{pmol}$ per mg beads. Heat treatment for $5 \mathrm{~min}$ at $75^{\circ} \mathrm{C}$ was performed for each cycle. 
other hand, $41 \%$ of the capture efficiency of the surface streptavidin remained after the three cycles of heat treatment. These results indicate that the addition of free PEG to the buffer solution only slightly improves the thermal stability of the immobilized streptavidin.

We next evaluated the change in the ssDNA capture ability of PEG/SA-MB after heat treatment. Figure 5 shows the change in the capture efficiency of biotinylated ssDNA according to the number of heat-treatment cycles. In this experiment, PEG/SA-MB and SA-MB, each possessing $26.4 \mathrm{pmol}$ streptavidin per mg beads, were used. To construct a PEG layer with sufficient density on the streptavidinimmobilized surface, we selected $0.2 \mu \mathrm{M}$ as the streptavidin concentration in the preparation step to leave some remaining surface open for consecutive PEG immobilization. The capture efficiencies were again defined as the percentages of the initial values (before heat treatment). These efficiencies decreased with an increasing number of heattreatment cycles. In the case of the SA-MB, only approximately $29 \%$ of the biotinylated ssDNA was captured $(O)$ after the SA-MB was heated three times. In contrast, in the case of the PEG/SA-MB, approximately $62 \%$ of the biotinylated ssDNA was captured $(\bullet)$, even after three cycles of heat treatment. These results clearly indicate that the co-immobilization of a PEG tethered-chain layer on the magnetic bead surfaces with streptavidin resulted in a great improvement in the thermal stability of the immobilized streptavidin.

\section{CONCLUSION}

This is the first report of the use of a co-immobilized PEG layer to prevent the heat-induced inactivation of the biotin-recognition ability of SA-MB; the co-immobilized PEG tethered-chain layer is an effective treatment for improving not only enzymatic activity ${ }^{12}$ but also the molecular recognition ability of a protein under high-temperature conditions. Furthermore, this study also revealed that the protective effect against the heat-induced inactivation of SA-MB of the coimmobilization of a PEG tethered-chain layer was more effective than that of the addition of free PEG in solution, indicating the importance of the polymer/protein hybrid surface in developing highperformance biomaterials. Recently, SA-MB has been widely used as bioseparation materials in diverse applications for biopanning, biomacromolecule isolation, immunoassays and genome sequencing. In these cases, SA-MB is often used in extreme conditions, such as high temperature, low $\mathrm{pH}$ and high-surfactant concentration. The modified PEG/SA-MB, displaying high thermal stability, would be expected to perform effectively in these extreme conditions.

\section{ACKNOWLEDGEMENTS}

This research was supported by a grant-in-aid for scientific research in priority areas, no. 19021007 (Life Surveyor), and a grant-in-aid for scientific research on innovative areas, no. 20106011 (Soft Interface), from the Ministry of Education, Science, Sports and Culture of Japan.

1 Sarkar, P., Pal, P. S., Ghosh, D., Setford, S. J. \& Tothill, I. E. Amperometric biosensors for detection of the prostate cancer marker (PSA). Int. J. Pharm. 238, 1-9 (2002).

2 Kaiser, T., Gudat, P., Stock, W., Pappert, G., Grol, M., Neumeier, D. \& Luppa, P. B. Biotinylated steroid derivatives as ligands for biospecific interaction analysis with monoclonal antibodies using immunosensor devices. Anal. Biochem. 282, 173-185 (2000).

3 Penalva, J., Puchades, R. \& Maquieira, A. Analytical properties of immunosensors working in organic media. Anal. Chem. 71, 3862-3872 (1999).

4 Lyon, L. A., Musick, M. D. \& Natan, M. J. Colloidal Au-enhanced surface plasmon resonance immunosensing. Anal. Chem. 70, 5177-5183 (1998).

5 Lee, J. H., Lee, H. B. \& Andrade, J. D. Blood compatibility of polyethylene oxide surfaces. Prog. Polym. Sci. 20, 1043-1079 (1995).

6 Katsumi, U., Yuki, H., Atsushi, T., Keitaro, Y., Shuji, K., Keichiro, Y., Ichiro, Y., Hidenori, O., Kazunori, K. \& Yukio, N. Creation of a mixed poly(ethylene glycol) tethered-chain surface for preventing the nonspecific adsorption of proteins and peptides. Biointerphases 2, 126-130 (2007).

7 Nagasaki, Y. PEG-b-polyamine stabilized bionanoparticles for nanodiagnostics and nanotherapy. Chem. Lett. 22, 564-569 (2008).

8 Yuan, X., lijima, M., Oishi, M. \& Nagasaki, Y. Structure and activity assay of nanozymes prepared by the coimmobilization of practically useful enzymes and hydrophilic block copolymers on gold nanoparticles. Langmuir 24, 6903-6909 (2008).

9 Yuan, X., Yoshimoto, K. \& Nagasaki, Y. High-performance immunolatex possessing a mixed-PEG/antibody coimmobilized surface: highly sensitive ferritin immunodiagnostics. Anal. Chem. 81, 1549-1556 (2009).

10 Yoshimoto, K., Nishio, M., Sugasawa, H. \& Nagasaki, Y. Direct observation of adsorption-induced inactivation of antibody fragments surrounded by mixed-PEG layer on a gold surface. J. Am. Chem. Soc. 132, 7982-7989 (2010).

11 Yuan, X., Fabregat, D., Yoshimoto, K. \& Nagasaki, Y. Efficient inhibition of interfacial nonspecific interaction to create practically utilizable high ferritin-response immunolatex. Anal. Chem. 81, 10097 (2009).

12 Nagasaki, Y., Yoshinaga, K., Kurokawa, K. \& lijima, M. Thermal- and dispersion-stable lipase-installed gold colloid: PEGylation of enzyme-installed gold colloid. Colloid Polym. Sci. 285, 563-567 (2007).

13 Waner, M. J., Navrotskaya, I., Bain, A., Oldham, E. D. \& Mascotti, D. P. Thermal and sodium dodecylsulfate induced transitions of streptavidin. Biophys. J. 87, 2701-2713 (2004).

14 Uchida, K., Otsuka, H., Kaneko, M., Kataoka, K. \& Nagasaki, Y. A reactive poly (ethylene glycol) layer to achieve specific surface plasmon resonance sensing with a high S/N ratio: the substantial role of a short underbrushed PEG layer in minimizing nonspecific adsorption. Anal. Chem. 77, 1075-1080 (2005).

15 Yoshimoto, K., Hirase, T., Nemoto, S., Hatta, T. \& Nagasaki, Y. Facile construction of sulfanyl-terminated poly(ethylene glycol)-brushed layer on a gold surface for protein immobilization by the combined use of sulfanyl-ended telechelic and semitelechelic poly(ethylene glycol)s. Langmuir 24, 9623-9629 (2008).

16 Satomi, T., Nagasaki, Y., Kobayashi, H., Otsuka, H. \& Kataoka, K. Density control of poly(ethylene glycol) layer to regulate cellular attachment. Langmuir 23, 6698-6703 (2007).

17 Andersson, E. \& Hahnhagerdal, B. Enzyme action in polymer and salt solutions .1. Stability of penicillin acylase in poly(ethylene glycol) and potassium phosphate solutions in relation to water activity. Biochim. Biophys. Acta 912, 317-324 (1987).

18 Welte, W., Leonhard, M., Diederichs, K., Weltzien, H. U., Restall, C., Hall, C. \& Chapman, D. Stabilization of detergent-solubilized $\mathrm{Ca}^{2+}$-ATPase by poly(ethylene glycol). Biochim. Biophys. Acta 984, 193-199 (1989).

19 Arakawa, T. \& Timasheff, S. N. Mechanism of polyethylene glycol interaction with proteins. Biochemistry 24, 6756-6762 (1985).

Supplementary Information accompanies the paper on Polymer Journal website (http://www.nature.com/pj) 